\title{
HIGHLIGHTS
}

HEPATITIS

\section{Genetic variant is associated with spontaneous clearance of HCV}

A single nucleotide polymorphism $3 \mathrm{~kb}$ upstream of the $I L 28 B$ gene is associated with spontaneous clearance of $\mathrm{HCV}$ in unselected populations, report Thomas et al. in Nature.

This polymorphism is strongly associated with a sustained virologic response to HCV treatment. "Thus, it seemed possible that this same variant might [also influence] spontaneous clearance of the virus," explains lead researcher, Mary Carrington.

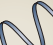
西 Carringto and colleagues genotyped 1,008 individuals from six independent $\mathrm{HCV}$ cohorts, 620 of whom had persistent infection;

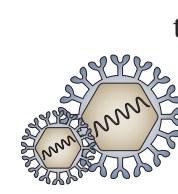

the other 388 had spontaneously cleared the virus. She points out that "the CC genotype at this locus [is] associated with resolution of $\mathrm{HCV}$ infection in individuals of European and African ancestry." Individuals with the CC genotype were three times more likely to clear the virus than those with the CT and TT genotypes combined.

"The frequency of the protective C allele varies remarkably across world populations," notes Carrington, whose team also analyzed genotyping data from 2,371 individuals from 51 populations.

IL28B encodes interleukin 28B

(also known as interferon $\lambda 3$ ), one of three type III interferons. These molecules, which are activated by

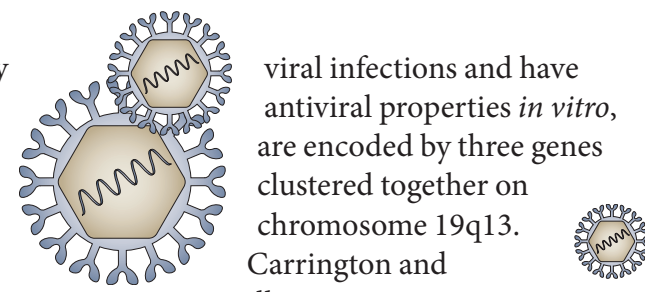

colleagues are now

investigating the molecular genetics of this locus to gain understanding of the function and evolution of these genes. In parallel, a phase II clinical trial is testing IL-29, one of the other type III interferons, as a treatment for HCV. "Maybe the protective form of IL-28B should be tested too," suggests Carrington.

Joana Osório

Original article Thomas, D. L. et al. Genetic variation in IL28B and spontaneous clearance of hepatitis $C$ virus. Nature 461, 798-801 (2009) 